\title{
Atmospheric Correction of Multiple Reflectance using S3
}

\author{
Dhruti Prajapati \\ College of Agricultural Information \\ Technology, AAU, Anand \\ dhruti.m.25@gmail.com
}

\author{
Dr. Mayur Raj \\ College of Agricultural Information \\ Technology, AAU, Anand \\ mraj@aau.in
}

\author{
Dr. Manoj Lunagariya \\ Department of Meteorology, BACA, \\ AAU, Anand \\ ml@aau.in
}

\begin{abstract}
In remote sensing multi-temporal images are used for assorted type of analysis. The image which is taken by satellite or airborne sensors is affected by atmosphere, so the reflected solar radiation of the image is not actual. Image with incorrect reflectance has no means. Such kind of image need to be corrected before usage. Image comprises of list of values, which needs to undergo this correction process. Existing paradigm provides a means to correct a single reflectance value at a time. Thus, for a list of values, this correction process is cumbersome and tedious. This paper presents a paradigm which allows to correct atmospheric correction of satellite signal for single as well as multiple reflectance values using S3 (Satellite Signal Simulator).
\end{abstract}

Keywords - 6S, Atmospheric Correction, Multiple Reflectance.

$* * * * *$

\section{I.INTRODUCTION}

For the development of new techniques or validating studies related to land cover dynamics remotely sensed images are useful. The imagery taken by satellites are mainly altered by the atmospheric particles due to absorption and scattering of the earth surfaces' radiation. Remotely sensed data is available with errors, so its utilization requires preprocessing[1]. These pre-processing comprises of removal of radiometric and geometric errors. In radiometric errors removal phase, after removing band and band level errors atmospheric errors are removed. The process of removing the effect of atmosphere from the remotely sensed data is called as Atmospheric correction.Atmospheric correction is used for retrieving the surface properties from remotely sensed data accurately after removing effects of atmosphere[2][3]. Atmospheric correction improves accuracy of image classification[4]. The radiative transfer model is utilized for examining clouds, other atmospheric components or surface features in the $3 \mathrm{D}$ radiative field as an analytic apparatus[5]. Various atmospheric corrections models based on radiative transfer code are available in market for example 6S[6], MODTRAN[7], COST, LOWTRAN [7], RCS, DOS simplified method for atmospheric correction (SMAC) [8].

The Second Simulation of the Satellite Signal in the Solar Spectrum (6S) is a Radiative Transfer Model widely used by remote sensing community[9][10], a highly accurate radiative transfer code, for a wide range of atmospheric conditions ) [8]. 6S was originally developed by a team led by Eric Vermote.In order to admonish natural hazards such as droughts, derivation of vegetation indices from satellite imageryby considering hydrometeorological data is of utmost importance.In its comparison even though
SMAC is 3000 time faster in processing SEVIRI scene it was found to error prone under vivid atmospheric conditions [8]. Jeffery and Alex introduced two solutionsfor radiative transfer problem. First solution was in from of Streamer tool capable of deriving radiance at surface leveland within atmosphere. This tool is used for developing remote-sensing algorithms. Second tool is FluxNet, it uses neural networkfor correction surface radiance [11].Atmospheric effects on satellite imagery must be eradicated before deriving any vegetation indices [12]. In case of a need for a speedy correction with relatively correct results, the $6 \mathrm{~S}$ modelling procedure with minimum input can be employed[4].

The system presented in this paper is basedon windows platform for retrieving true surface reflectance values of earth surface from remotely sensed satellite image. Correction of Multiple reflectance values are useful for producing Lookup Table. This lookup table is further useful for the correction of satellite image. From this lookup table we can correct any pixel value within no time.

\section{METHODOLOGY}

For atmospheric correction of satellite data, the $6 \mathrm{~S}$ model needs parameters for atmospheric conditions, geometrical conditions, target and sensor altitude, spectral condition, ground reflectance etc.

With the existing system the user needs to create an Input file containing these parameters for atmospheric correction. Input parameter consist of information pertaining to Atmospheric Profile (atmospheric model type, radiosonde measurements, amount of water vapour and ozone), Aerosol Profiles, Ground Reflectance, Geometries, Altitudes, Wavelengths, Atmospheric Corrections[13].During 
calculation of single reflectance value, based on these input parameters output put file is generated. Users' can use a latest version of web based $6 \mathrm{~S}$ calculator for rectifying radiance value free. But it is limited to single value and session [14]. GRASS also provide inbuilt utility for atmospheric correction which ultimately uses $6 \mathrm{~S}$ in background.

In normal scenario as per the available options for calculating atmospheric correction for a range/list of reflectance value, above process should be repeated for each single reflectance value. Thus, for multiple reflectance values user needs to do manually editing of the input file and for each reflectance value user must repeat this process which is time consuming, error proneand tedious process. For atmospheric correction of a satellite image the user takes unique reflectance values of an image and follow the abovementioned procedure. Online facility is avail for deriving single reflectance value which is comparatively slower depending upon connectivity and speed of internet.

The $\mathrm{S} 3$ is an interface to 6S. It is developed using DOTNET ambiance by using $\mathrm{C \#}$ language. This system is standalone and doesn't require internet connection. A standalone window-based system provided easy user interface for calculation of reflectance value for single, multiple or range and even for image. It uses $6 \mathrm{~S}$ V1.1 executable component. For the processing of a satellite image it uses GDAL libraries. It allows users to run $6 \mathrm{~S}$ simulations by interactive graphical user interface rather than dealing with cryptic $6 \mathrm{~S}$ input and output files.

\section{Procedure for generating Lookup Table}

- Collect all the values for important parameters which are used for correction of image.

- Now considering these parameters iterate over all the values of an image i.e. reflectance values.

- Now for all possible combination we have atmospherically corrected reflectance.

- Update image vector or store it into a table along with parameters for future reference[15].

\section{RESULT AND DISCUSSION}

\section{Activity Diagram}

First step in activity diagram [Fig-1] indicates that it is initiated by user by entering various parameters. Before generating input file and extracting correct reflectance value these parameters are validated. A new output file is created to store correction. For correction of single reflectance value process will end here. In case of correction of list/range of reflectance value, corrected value will become part of lookup table.

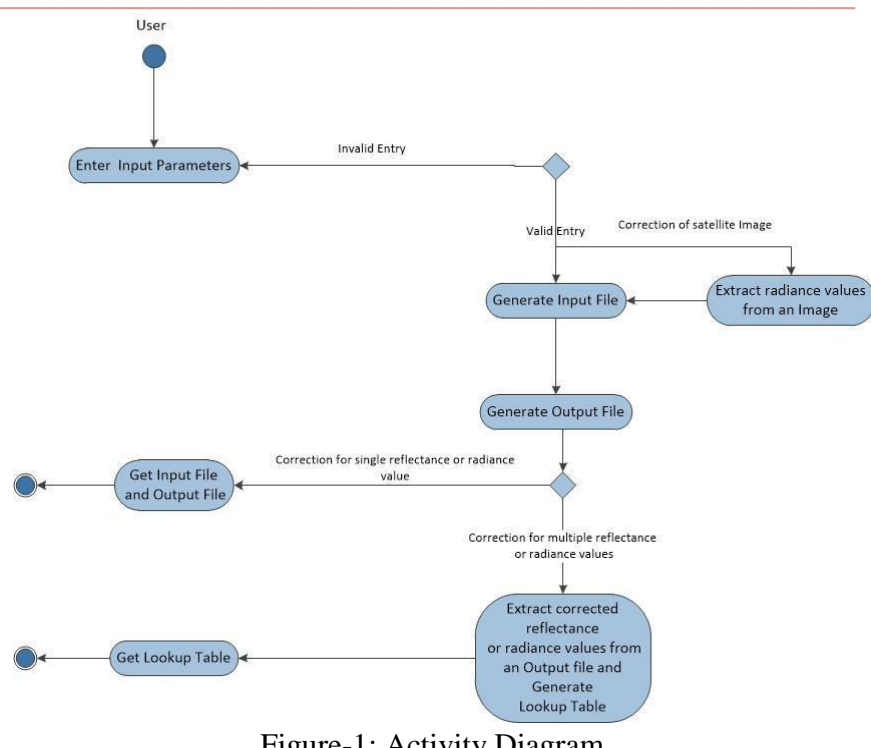

Figure-1: Activity Diagram

The home screen or dashboard [Fig-2] of the system which allows user to select Input parameter for which he/she needs to enter or select some proposed standard condition or define his/her own conditions. The sequence of selecting section for data entry or selecting values for Input parameter is not mandatory. User can enter it in any sequence, but all the parameters are mandatory.

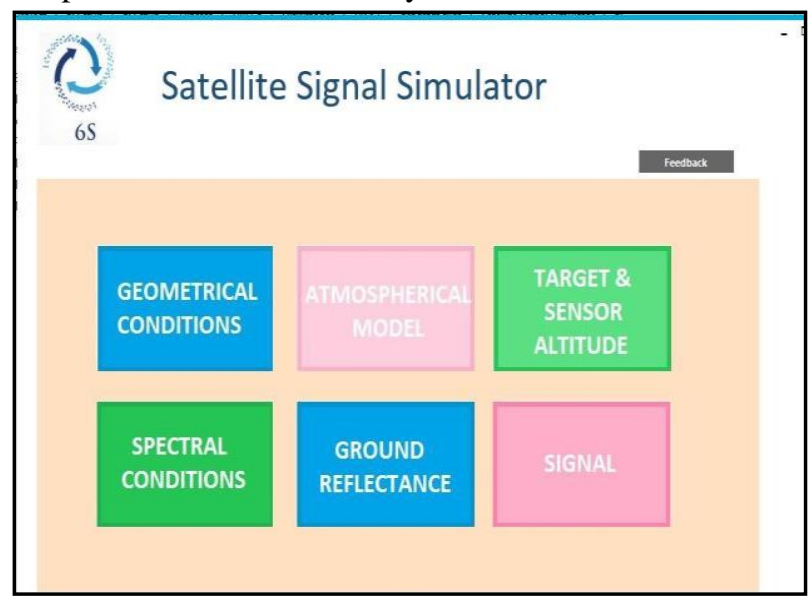

Figure 2 - Dashboard

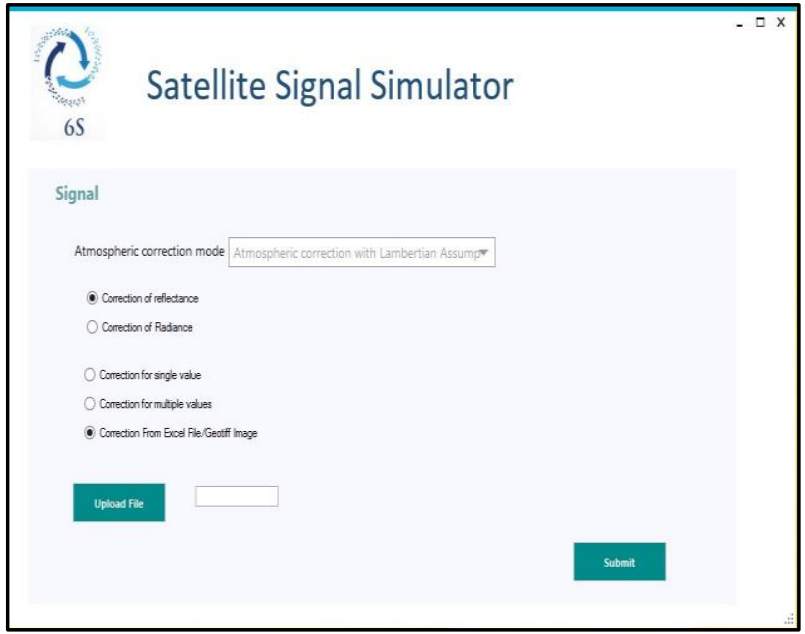

Figure 3 - Input values for Atmospheric correction or satellite imagery 


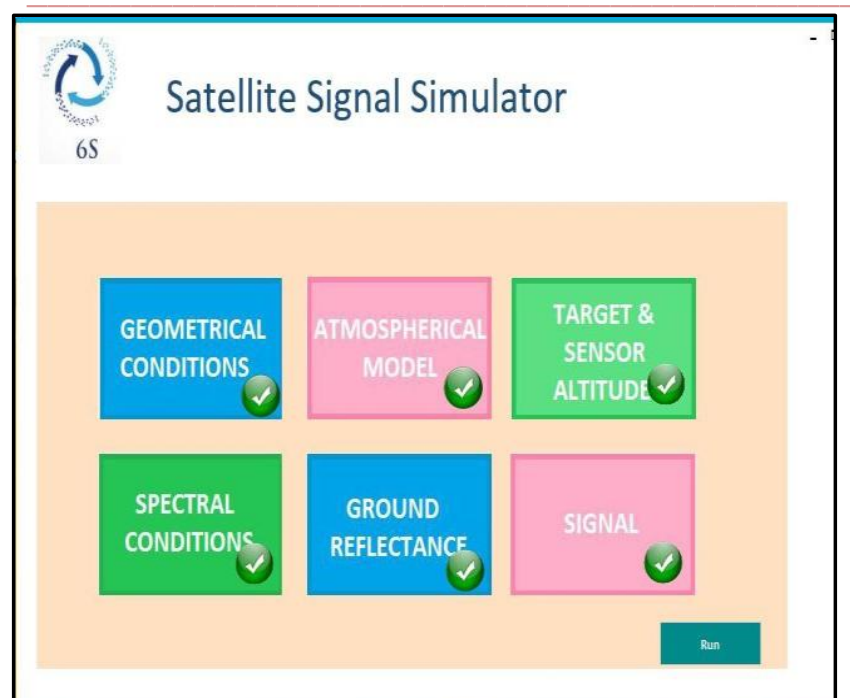

Figure4- Validated Input values for Atmospheric correction

In [Fig-3], user needs to select Signal parameter conditions for which he/she needs atmospheric correction. Based on selected condition system allows user to enter values or to upload a file. After user stores valid values for each input parameter of a section, it will be updated with tick on it as shown in [Fig-4]. Now S3is ready and user can start simulation.

Below [Fig-5] is the screen which shows output of correction of multiple reflectance values for a given range of surface reflectance values. There is also option available to store this result in Excel file. By clicking on Export to Excel button the result table will be saved into an Excel file.

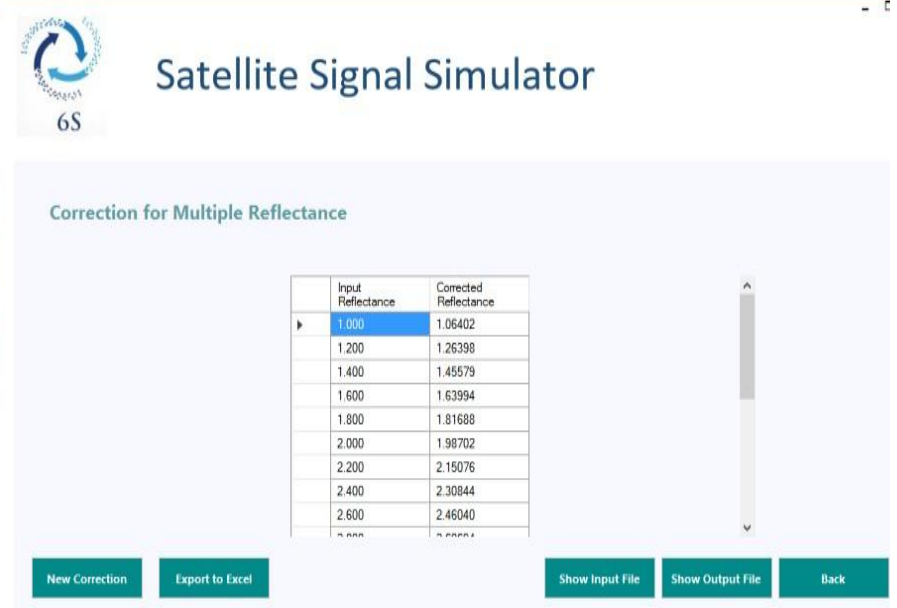

Figure 5 - Validated Input values for Atmospheric correction

The presented system eliminates the process of manually editing Input files and provides aoffline graphical user interface to the $6 \mathrm{~S}$ model. The system makes easier to use $6 \mathrm{~S}$ and adds new features:

- The ability to run many simulations easily and quickly.

- The system provides following options for atmospheric correction.

- Correction of single reflectance or radiance value.
- Correction of reflectance or radiance values for a specific range given by the user.

- Correction of the reflectance or radiance values from an Excel sheet uploaded by the user.

\section{CONCLUSION}

Compared to single value correction of Atmospheric errorsin space borne remote sensing observations, multiple value options, reduces time and efforts required to correct satellite imagery.S3 is alightweight, standalone, userfriendly windows-based tool, itneither require an internet connection for performing atmospheric correction nor even extra software like GRASS. An additional utility or next stage can be a corrected image based on $6 \mathrm{~S}$ atmospheric correction model, an ultimate result expectation for end users.

\section{References}

[1] M. Emimal and P. Dr. Kannan, "Classification of Remote Sensing Images using Wavelet Based Contourlet Transform and Accuracy Analysis of Classified Images," Int. J. Advanced Networking and Applications, vol. 9, no. 5, pp. 3601-3606, 2018.

[2] R. Holm, R. Jackson, B. Yuan, M. Moran, P. Slater and S. Bigger, "Surface reflectance factor retrieval from Thematic Mapper data," Remote Sensing of Environment, p. 27:47-57, 1989.

[3] M. Moran, R. Jackson, P. Slater and P. Teillet, "Evaluation of simplified procedures for retrieval of land surface reflectance factors from satellite sensor output," Remote Sensing of Environment, vol. 41, p. 169-184, 1992.

[4] A. S. Mahiny and B. J. Turner, "A Comparison of Four Common Atmospheric Correction Methods," PHOTOGRAMMETRIC ENGINEERING \& REMOTE SENSING, vol. 73, no. 4, p. 361-368, April 2007.

[5] W. O'HIROK and C. GAUTIER, "A Three-Dimensional Radiative Transfer Model to Investigate the Solar Radiation within a Cloudy Atmosphere. Part I: Spatial Effects," JOURNAL OF THE ATMOSPHERIC SCIENCES, vol. 55, pp. 2162-2179, 1998.

[6] E. F. Vermote, D. Tanre, J. L. Deuze, M. Herman and J. J. Morcrette, "Second Simulation of the Satellite Signal in the Solar Spectrum, 6S: An Overview," IEEE Transactions on Geoscience and Remote Sensing, vol. 35, no. 3, pp. 675-686, 1997.

[7] B. Y. Wu, W. Li, H. B. Chen, F. Li, W. X. Zhang and D. Lu, "Practical Algorithms of Atmospheric Radiative Transfer," Meteorological Press, Beijing, pp. 21-40, 1998.

[8] S. R. Proud, R. Fensholt, M. O. Rasmussen and I. Sandholt, "A comparison of the effectiveness of $6 \mathrm{~S}$ and SMAC in correcting for atmospheric interference of Meteosat Second Generation images," JOURNAL OF GEOPHYSICAL RESEARCH, vol. 115, pp. 1-14, 10 September 2010.

[9] Y. Zhang, X. Wang and Y. Chen, "An Improved 6S Code for Atmospheric Correction Based on Water Vapor Content," Advances in Remote Sensing (Scientific Research), vol. 1, pp. 14-18, 2012.

[10] J. Martin, F. Eugenio, J. Marcello, A. Medina and J. A. Bermejo, "Atmospheric correction models for high resolution 
WorldView-2 multispectral imagery: A case study in Canary Islands, Spain.," in SPIE - The International Society for Optical Engineering, November 2012.

[11] J. R. Key and A. J. Schweiger, "Tools for atmospheric radiative transfer: Streamer and FluxNet," Computers \& Geosciences, ELSEVIER, vol. 24, no. 5, pp. 443-451, 1998.

[12] H. D. G., G. Papadavid, A. Agapiou, K. Themistocleous, M. G. Hadjimitsis, A. Retalis, S. Michaelides, N. Chrysoulakis, L. Toulios and C. R. I. Clayton, "Atmospheric correction for satellite remotely sensed data intended for agricultural applications: impact on vegetation indices," Natural Hazards and Earth System Sciences, vol. 10, p. 89-95, 2010.

[13] "Parameter Setting Py6S 1.7.2 Documentation," Robin Wilson Revision 0ee60d56., 2012. [Online]. Available: https://py6s.readthedocs.io/en/latest/params.html. [Accessed 10 April 2019]. \begin{tabular}{cccc} 
[14] “6SV & Home," & [Online]. & \multicolumn{2}{c}{ Available: } \\
http://6s.ltdri.org/pages/run6SV.html. [Accessed & 24 & June
\end{tabular} 209].

[15] R. Wilson, "Can I atmospherically-correct my images with Py6S? - Robin's Blog," 3 March 2013. [Online]. Available: http://blog.rtwilson.com/can-i-atmospherically-correct-myimages-with-py6s/. [Accessed 04 April 2019]. 